\title{
Análisis Clínico e Histomorfológico de la Mucosa Oral Normal, Hiperplasia Fibroepitelial Inflamatoria Oral y Displasia Epitelial Oral
}

\author{
Clinical and Histomorphological Analysis of Normal Oral Mucosa, \\ Oral Inflammatory Hyperplasia and Oral Epithelial Displasia
}

\begin{abstract}
"Manuel Antonio Gordón-Núñez; ${ }^{* *}$ Francisco Lopes da Silva Júnior; ${ }^{* * *}$ Hévio Freitas de Lucena; ${ }^{* * * *}$ Hébel Cavalcanti Galvão; ${ }^{* * * *}$ Lélia Batista De Souza \& ${ }^{* * * *}$ Leão Pereira Pinto
\end{abstract}

GORDÓN-NÚÑEZ, M. A.; SILVA JÚNIOR F. L.; LUCENA, H. F.; GALVÃO, H. C.; DE SOUZA, L. B. \& PEREIRA PINTO, L. Análisis clínico e histomorfológico de la mucosa oral normal, hiperplasia fibroepitelial inflamatoria oral y displasia epitelial oral. Int. J. Morphol., 26(2):345-352, 2008.

RESUMEN: El desarrollo del cáncer oral puede darse a partir de una mucosa aparentemente normal o a partir de una lesión precursora inicial, las cuales pueden representar pasos diferentes del proceso de carcinogénesis epitelial oral. El objetivo de este estudio fue analizar morfológicamente, a través de la técnica de la hematoxilina / eosina, la mucosa oral normal, hiperplasia fibroepitelial oral y displasia epitelial oral, con el propósito de obtener mayor información sobre las alteraciones epiteliales que pueden preceder al establecimiento y evolución de una neoplasia propiamente tal. Fue constatado que muchas de las alteraciones morfológicas observadas en las hiperplasias fibroepiteliales orales probablemente eran asociadas al proceso inflamatorio, donde es conocida la acción de mediadores químicos con capacidad de promover modificaciones morfológicas celulares y en la estructura tisular y no necesariamente representan alteraciones comunes de lesiones potencialmente malignas. Correlacionando el aspecto clínico de las lesiones con el diagnóstico histopatológico de displasia epitelial oral, no fue observada diferencia expresiva; sin embargo, la mayoría de los especimenes tuvo diagnostico clínico de leucoplasia. Se concluyó que la simple observación de las características morfológicas de las alteraciones de la mucosa oral puede ayudar el establecimiento del diagnóstico de las lesiones sufridas por la mucosa; sin embargo, no ofrecen mucha información sobre cuáles serían los eventos que llevarían al establecimiento de una determinada lesión.

PALABRAS CLAVE: Mucosa oral; Hiperplasia inflamatoria oral; Displasia epitelial oral; Histomorfología.

\section{INTRODUCCIÓN}

Alteraciones en el equilibrio homeostático del epitelio de la mucosa oral por algún factor agresor, pueden promover el desarrollo de lesiones como la hiperplasia fibroepitelial inflamatoria oral(HFIO), la displasia epitelial oral (DEO) o, en los casos más graves, a neoplasias epiteliales (González-Moles, 1997; Montenegro \& Franco, 1999).

La hiperplasia representa el aumento de tamaño de un tejido o órgano debido al mayor número de células, proceso que ocurre de forma exclusiva en tejidos con capacidad mitótica, comprometiendo células biológicamente normales capaces de responder a estímulos sin perder su patrón morfológico y con preservación de la membrana basal (Zerdoner, 2003; Kumar et al., 2005).

Generalmente, las alteraciones epiteliales de la HFIO son acompañadas por cambios a nivel subepitelial, tales como, hipervascularización, fibrosis e infiltrado inflamatorio predominantemente crónico (Kambic, 1997). Estas lesiones son constantemente asociadas con factores irritantes locales crónicos de baja intensidad y han sido el foco de interés de muchos estudios debido a la posibilidad de que puedan constituir entidades pre malignas; sin embargo, ese asunto es controversial (Zerdoner).

M.D, Ph.D. en Patología Oral. Alumno del Programa de Postdoctorado IFARHU / SENACYT Panamá/UFRN, Brasil.

** Académico de Odontología, Universidad Potiguar - UnP, Brasil.

**** Biólogo, Especialista en Bioquímica, Técnico del Laboratorio de Imuno-histoquímica del Programa de Postgrado en Patología Oral de la Universidad Federal de Rio Grande del Norte - UFRN, Brasil.

***** Prof., Dr., M.D., Ph.D. del Programa de Postgrado en Patología Oral de la Universidad Federal de Rio Grande del Norte - UFRN, Brasil. Fragmento de tesis financiada por el Consejo Nacional de Desarrollo Científico y Tecnológico - CNPq, Brasil. 
El término displasia epitelial agrupa diferentes cambios estructurales del epitelio caracterizados por la combinación variable de fenómenos histológicos indicadores de un desorden de la maturación y proliferación celulares, donde la gravedad de la lesión depende del mayor o menor número de trastornos epiteliales verificados (González-Moles; Castellanos, 2002). La DEO no es asociada a ningún aspecto clínico específico; sin embargo, las leucoplasias y eritroplasias son lesiones clásicamente relacionadas a alteraciones histopatológicas de displasias, las cuales asocian a la DEO con un alto riesgo de transformación maligna (Reibel, 2003).

Diversos factores han sido relacionados con la etiología de las DEOs, como el consumo crónico de alcohol y tabaco (Jaber et al., 1999; Jaber et al., 2003) y agentes biológicos (McCullogh et al., 2002). La Organización Mundial de la Salud (OMS) preconizó criterios histomorfológicos que deben ser considerados en el diagnóstico de esas lesiones (Pindorg et al., 1997).

La gravedad de la DEO puede ser clasificada, de acuerdo con Neville et al. (2004) en: displasia leve, moderada e intensa. Debido a la subjetividad de los criterios de evaluación establecidos para la clasificación de la displasia, no existe un sistema de graduación ideal que proporcione resultados consistentes y reproducibles (Van der Waal et al., 1997).

La transición de MON para carcinoma epidermoide es un proceso multifactorial extremamente complejo, lo que justifica los numerosos estudios que intentan identificar las alteraciones en la mucosa oral normal que preceden a la malignización. El desarrollo de algunos tipos de lesiones puede representar pasos diferentes en ese proceso (Küffer \& Lombardi, 2002).

Basado en la literatura consultada, este estudio tuvo como objetivo realizar análisis clínico e histomorfológico de especímenes de mucosa oral normal, hiperplasia fibroepitelial inflamatoria oral y displasia epitelial oral, con el objetivo de obtener mayores informaciones que ayuden a entender la complejidad de las alteraciones sufridas por el epitelio de la mucosa oral que pudiesen preceder a la instalación de una neoplasia.

\section{MATERIAL Y MÉTODO}

Fueron evaluados 11 especímenes de mucosa oral normal (MON), 16 de hiperplasia fibroepitelial inflamatoria oral (HFIO) y 25 de displasia epitelial oral (DEO), existentes en los archivos del Servicio de Anatomía Patológica de la Disciplina de Patología Oral del Departamento de Odontología de la Universidad Federal de Rio Grande del Norte, Brasil.

Los datos de los pacientes e informaciones referentes a los especímenes fueron colectados de los expedientes clínicos. Fue realizado un análisis histomorfológico bajo microscopía de luz, con cortes de $5 \mu \mathrm{m}$ de grosor, teñidos a través de la técnica de la hematoxilina y eosina. Las DEOs fueron diagnosticadas llevando en consideración aspectos histomorfológicos preconizados por la Organización Mundial de la Salud (OMS) (Pindborg et al.) y clasificadas de acuerdo al sistema propuesto por Neville et al.

Este estudio fue aprobado por el Comité de Ética en Investigación de la UFRN, a través del protocolo $\mathrm{N}^{\circ} 37 / 05$ y por el Sistema Nacional de Ética en Investigación-SISNEP 056280 .

\section{RESULTADOS}

Fueron evaluados 52 especímenes, de los cuales, 11 $(21,2 \%)$ eran de mucosa oral normal (MON), $16(30,8 \%)$ de hiperplasia fibroepitelial inflamatoria oral (HFIO) y 25 $(48,1 \%)$ de displasias epiteliales orales (DEO), siendo de éstas, $16(64,0 \%)$ leves, $2(8,0 \%)$ moderadas y $7(28,0 \%)$ intensas.

La Tabla I exhibe la distribución de la muestra en relación al tipo de espécimen, sexo, color de la piel y edad

Tabla I. Distribución de la muestra con relación al tipo de especimen, sexo, color de la piel y edad de los pacientes. Natal / RN, 2007.

\begin{tabular}{|c|c|c|c|c|c|c|c|c|c|c|c|c|c|c|c|c|c|c|c|}
\hline \multirow{3}{*}{$\begin{array}{c}\text { Tipo de } \\
\text { Especimen }\end{array}$} & \multicolumn{6}{|c|}{ Sexo } & \multicolumn{10}{|c|}{ Color de la piel del paciente } & \multicolumn{3}{|c|}{ Edad } \\
\hline & \multicolumn{2}{|c|}{ Masculino } & \multicolumn{2}{|c|}{ Femenino } & \multicolumn{2}{|c|}{ Total } & \multicolumn{2}{|c|}{ Leuco* } & \multicolumn{2}{|c|}{ Melano* } & \multicolumn{2}{|c|}{ Feod $^{*}$} & \multicolumn{2}{|c|}{$\mathrm{S} / \mathbf{I}^{*}$} & \multicolumn{2}{|c|}{ Total } & \multirow{2}{*}{$\mathbf{n}$} & \multirow{2}{*}{$\begin{array}{l}\text { Media + } \\
\text { DP }\end{array}$} & \multirow{2}{*}{ Min - Max } \\
\hline & $\mathbf{N}$ & $\%$ & $\mathbf{N}$ & $\%$ & $\mathbf{n}$ & $\%$ & $\mathbf{n}$ & $\%$ & $\mathbf{n}$ & $\%$ & $\mathbf{n}$ & $\%$ & $\mathbf{n}$ & $\%$ & $\mathbf{n}$ & $\%$ & & & \\
\hline $\mathrm{MON}^{*}$ & 3 & 27,3 & 8 & 72,7 & 11 & 21,2 & 6 & 254,5 & 1 & 9,1 & 2 & 20,0 & 2 & 18,2 & 11 & 21,2 & 11 & $48,36 \pm$ & $15,00-70,00$ \\
\hline HFIO $^{*}$ & 3 & 18,8 & 13 & 81,2 & 16 & 30,8 & 6 & 37,5 & 4 & 25,0 & 2 & 12,5 & 4 & 25,0 & 16 & 30,8 & 13 & 46,92 & $7,00-70,00$ \\
\hline $\mathrm{DEO}^{*}$ & 9 & 36,0 & 16 & 64,0 & 25 & 48,1 & 11 & 44,0 & 7 & 28,0 & 6 & 24,0 & 1 & 4,0 & 25 & 48,1 & 24 & 51,41 & $17,00-81,00$ \\
\hline Total & 15 & 28,8 & 37 & 71,2 & 52 & 100 & 23 & 44,2 & 12 & 23,1 & 10 & 19,2 & 7 & 13,5 & 52 & 100 & & & \\
\hline
\end{tabular}

MON: Mucosa oral normal; HFIO: Hiperplasia fibroepitelial inflamatoria oral; DEO: Displasia epitelial oral; Leuco: Leucoderma; Melano: Melanoderma; Feod: Feoderma; S/I: Sin información. 
GORDÓN-NÚÑEZ, M. A.; SILVA JÚNIOR F. L.; LUCENA, H. F.; GALVÃO, H. C.; DE SOUZA, L. B. \& PEREIRA PINTO, L. Análisis clínico e histomorfológico de la mucosa oral normal, hiperplasia fibroepitelial inflamatoria oral y displasia epitelial oral. Int. J. Morphol., 26(2):345-352, 2008.

de los pacientes. No fue posible obtener información referente a la edad de 2 pacientes con HFIO y de uno con DEO. La distribución de los especímenes de MON, HFIO y DEO con relación a la localización anatómica, se encuentra en la Tabla II. En la Tabla III se presenta la distribución de las muestra de MON, HFIO y DEO, con relación a los diagnósticos clínicos presentes en las fichas de los especímenes evaluados en el estudio.

En la evaluación histomorfológica realizada en los cortes teñidos a través de la técnica de hematoxilina/eosina y analizados bajo microscopía de luz, fue observado que los especímenes de MON presentaron las características descritas por Stern (Bhaskar,1989), o sea un revestimiento epitelial estratificado paraqueratinizado (9 especímenes $81,8 \%$ ) e hiperparaqueratinizado ( 2 especímenes $-18,2 \%$ ); la lámina propia subyacente era constituida por un tejido conectivo fibroso de densidad variable, siendo denso en 7 especímenes $(63,6 \%)$ y laxo en $4(36,4 \%)$, tal tejido era moderadamente vascularizado exhibiendo, ocasionalmente, discreto infiltrado inflamatorio mononuclear en posición predominantemente perivascular (Fig. 1).

Todos los especímenes de HFIO estaban revestidos por un epitelio estratificado escamoso paraqueratinizado (8 especímenes $50,0 \%$ ), hiperparaqueratinizado ( 5 especímenes $-31,3 \%$ ) y ortoqueratinizado (3 especímenes $-18,8 \%$ ), exhibiendo hiperplasia, degeneración hidrópica, espongiosis, discreta exocitosis en puntos focales de 6 especímenes $(37,5 \%)$ y discreto a moderado infiltrado inflamatorio mononuclear en $10(62,5 \%)$ y $6(37,5 \%)$ especímenes, respectivamente. La lámina propia era constituida por tejido conectivo denso en 12 especímenes $(75,0 \%)$ y tejido conectivo laxo en 4 casos $(25,0 \%)$ (Figs. 2 y 3$)$.

Los especímenes de DEO exhibieron las características histomorfológicas preconizadas por la OMS, verificaándose un revestimiento epitelial estratificado paraqueratinizado (6 especímenes $-24,0 \%$ ), hiperparaqueratinizado (14 especímenes - 56,0\%) y ortoqueratinizado (5 especímenes $-20,0 \%$ ). Todos los especímenes exhibieron hiperplasia de la camada basal, pleomorfismos celular y nuclear, pérdida de la relación núcleo-citoplasma, hipercromacia, áreas de pérdida de la estratificación (DEOs moderadas/intensas) y focos de exocitosis. Áreas con pérdida de la integridad de la membrana basal fueron constatadas en 9 especímenes $(36,0 \%)$.

La lámina propia estaba constituida por un tejido conectivo, cuya densidad variaba de fibroso denso (6 especímenes $-24,0 \%$ ) a laxo (19 especímenes $-76,0 \%)$, moderadamente vascularizado, sede de infiltrado inflamato- rio mononuclear de densidad variable, siendo predominantemente intenso en 6 especímenes $(24,0 \%)$, moderado en 16 $(64,0 \%)$ y discreto en 3 casos $(12,0 \%)$ (Figuras 4,5 y 6).

\section{DISCUSIÓN}

La integridad y funcionalidad del epitelio de la mucosa oral son garantizadas por un proceso de renovación constante, en el cual sus camadas o estratos se reorganizan estructuralmente como consecuencia de las modificaciones bioquímicas y morfológicas celulares (Winning \& Towsend, 2000).

En virtud de la acción de algún factor agresor puede darse un desequilibrio del proceso homeostático tisular, incluso, las células epiteliales pueden experimentar alteraciones bioquímicas y morfológicas. Sin embargo, éstas pueden diferir de aquellas comunes al patrón de normalidad característico de la mucosa oral. Siendo así, en algunos casos, dependiendo del tipo e intensidad de la agresión sufrida por el epitelio, sus células pueden adaptarse progresivamente o sufrir un proceso regresivo e incluso evolucionar a la muerte, dando lugar a las lesiones hiperplásicas, displásicas o, en los casos más graves, a las llamadas neoplasias epiteliales (Montenegro \& Franco; González-Moles).

El epitelio de la MON constituye un tejido con intensa actividad mitótica, respondiendo a una adaptación celular frente a la acción de agentes agresores crónicos de baja intensidad, tal epitelio puede sufrir un aumento del tamaño tisular producido por el número aumentado de células, comprometiendo su homeostasis funcional y consecuentemente llevar a una HFIO (Zerdoner).

La caracterización de la muestra de HFIO evaluada en este estudio, no difirió de los datos de la literatura (PintoCoelho \& Zucoloto, 1998), ya que no fue verificada diferencia en la ocurrencia de esa lesión con relación al sexo, edad ni color de piel de los pacientes. Los datos del estudio también concuerdan con la literatura consultada en lo referente a la localización más frecuente de las HFIOs, o sea, aquellas regiones de la mucosa oral que son expuestas frecuentemente a irritación o trauma. Tosios et al. (1998) observaron resultados similares a los de este estudio, ya que las HFIOs por ellos analizadas ocurrieron en regiones como lengua, labios, mucosa yugal y gingiva.

Es sugerida la posibilidad que las HFIOs representen lesiones potencialmente malignas; sin embargo, concordamos con Zerdoner sobre el carácter cuestionable de este asunto. 
Tabla II. Distribución de las muestras de mucosa oral normal (MON), hiperplasia fibroepitelial inflamatoria oral (HFIO) y displasia epitelial oral (DEO) con relación a la localización anatómica de los especímenes. Natal / RN, 2007.

\begin{tabular}{|c|c|c|c|c|c|c|}
\hline \multirow{3}{*}{$\begin{array}{c}\text { Localización } \\
\text { anatómica }\end{array}$} & \multicolumn{6}{|c|}{ Tipo de especimen } \\
\hline & \multicolumn{2}{|c|}{$\mathrm{MON}^{*}$} & \multicolumn{2}{|c|}{ HFIO $^{*}$} & \multicolumn{2}{|c|}{ DEO $^{*}$} \\
\hline & $\mathbf{n}$ & $\%$ & $\mathbf{n}$ & $\%$ & $\mathbf{N}$ & $\%$ \\
\hline Labio inferior & 3 & 30,0 & 0 & 0,0 & 4 & 16,0 \\
\hline Lábio superior & 0 & 0,0 & 1 & 6,25 & -- & 0,0 \\
\hline Paladar duro & 1 & 10,0 & 2 & 12,5 & 1 & 4,0 \\
\hline Paladar suave & 0 & 0,0 & -- & 0,0 & 1 & 4,0 \\
\hline Región retromolar & 2 & 20,0 & -- & 0,0 & 2 & 8,0 \\
\hline Piso oral & 1 & 10,0 & -- & 0,0 & -- & 0,0 \\
\hline Gingiva & 1 & 10,0 & 4 & 25,0 & 3 & 12,0 \\
\hline Mucosa yugal & 1 & 10,0 & 2 & 12,5 & 6 & 24,0 \\
\hline Fornice vestibular & 0 & 0,0 & 5 & 31,25 & -- & 0,0 \\
\hline Lengua & 0 & 0,0 & 1 & 6,25 & 4 & 16,0 \\
\hline Freno labial & 0 & 0,0 & -- & 0,0 & 1 & 4,0 \\
\hline Reborde alveolar & 1 & 10,0 & 1 & 6,25 & 3 & 12,0 \\
\hline Total & 10 & 19,61 & 16 & $\mathbf{3 1 , 3 7}$ & 25 & 49,02 \\
\hline
\end{tabular}

Tabla III. Distribución de las muestras de mucosa oral normal (MON), hiperplasia fibroepitelial inflamatoria oral (HFIO) y displasia epitelial oral (DEO) con relación a los diagnósticos clínicos registrados en las fichas de los especímenes. Natal / RN, 2007.

\begin{tabular}{|c|c|c|c|c|c|c|}
\hline \multirow{3}{*}{ Localización anatómica } & \multicolumn{6}{|c|}{ Tipo de especimen } \\
\hline & \multicolumn{2}{|c|}{ MON } & \multicolumn{2}{|c|}{ HFIO } & \multicolumn{2}{|c|}{ DEO } \\
\hline & $\mathbf{n}$ & $\%$ & $\mathbf{n}$ & $\%$ & $\mathbf{n}$ & $\%$ \\
\hline Hiperplasia fibroepit, inflam, oral & 8 & 73,0 & 13 & 82,0 & 4 & 16,0 \\
\hline Mucocele & 1 & 9,0 & 1 & 6,0 & --- & 0,0 \\
\hline Nevus melánico & 1 & 9,0 & --- & --- & --- & 0,0 \\
\hline Fibroma & 1 & 9,0 & --- & --- & --- & 0,0 \\
\hline Gingiva & --- & --- & 1 & 6,0 & --- & 0,0 \\
\hline Fibroma / hiperqueratosis & --- & --- & 1 & 6,0 & --- & 0,0 \\
\hline Leucoplasia & --- & --- & --- & --- & 7 & 28,0 \\
\hline Carcinoma & --- & --- & --- & --- & 3 & 12,0 \\
\hline Queilitis actínica & --- & --- & --- & --- & 3 & 12,0 \\
\hline Papiloma / fibroma & --- & --- & --- & --- & 1 & 4,0 \\
\hline Lipoma & --- & --- & --- & --- & 1 & 4,0 \\
\hline Hiperqueratosis / leucoplasia & --- & --- & --- & --- & 1 & 4,0 \\
\hline Hiperplasia gingival dilantínica & --- & --- & --- & --- & 1 & 4,0 \\
\hline Granuloma piogénico & --- & --- & --- & --- & 1 & 4,0 \\
\hline Liquen plano/leucoplasia & --- & --- & --- & --- & 1 & 4,0 \\
\hline Eritroplasia & --- & --- & --- & --- & 1 & 4,0 \\
\hline Sin información & --- & --- & --- & --- & 1 & 4,0 \\
\hline Total & 10 & 19,61 & 16 & 31,37 & 25 & 49,02 \\
\hline
\end{tabular}




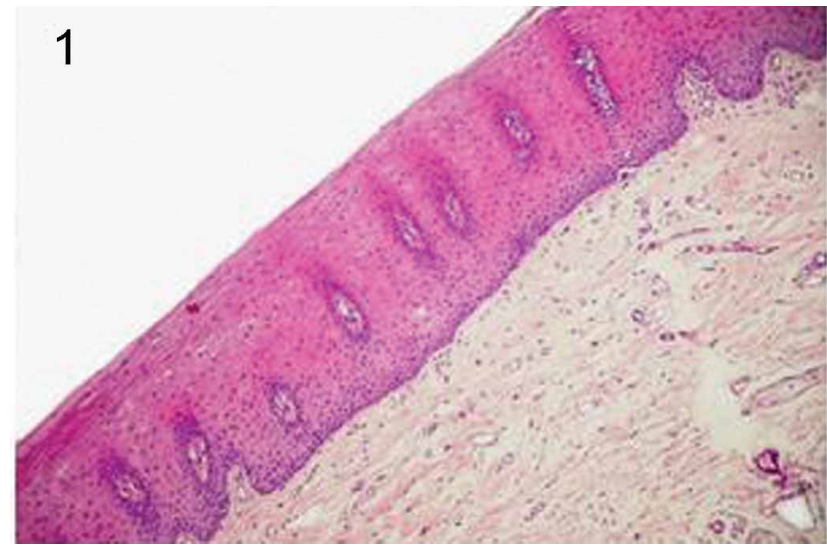

Fig. 1. Mucosa oral normal (MON) (H/E - 100x).

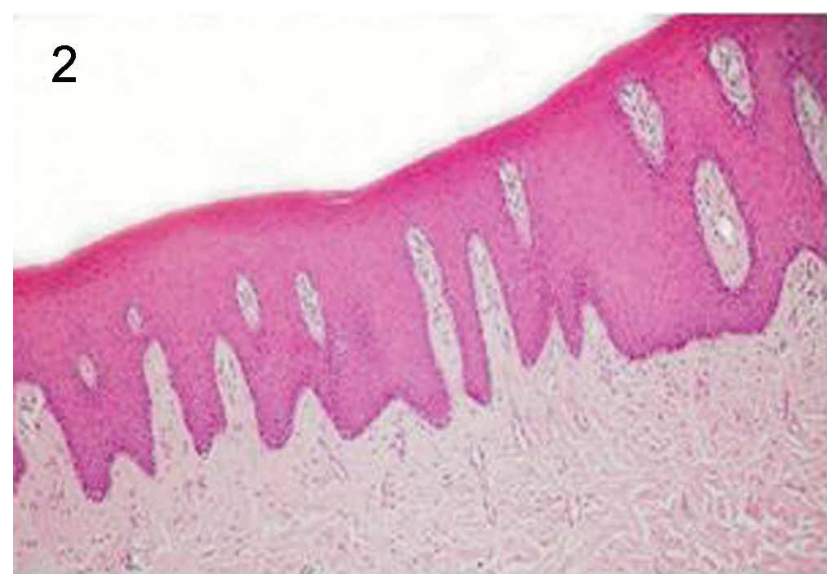

Fig. 2. Hiperplasia fibroepitelial inflamatoria oral (H/E - 100x).

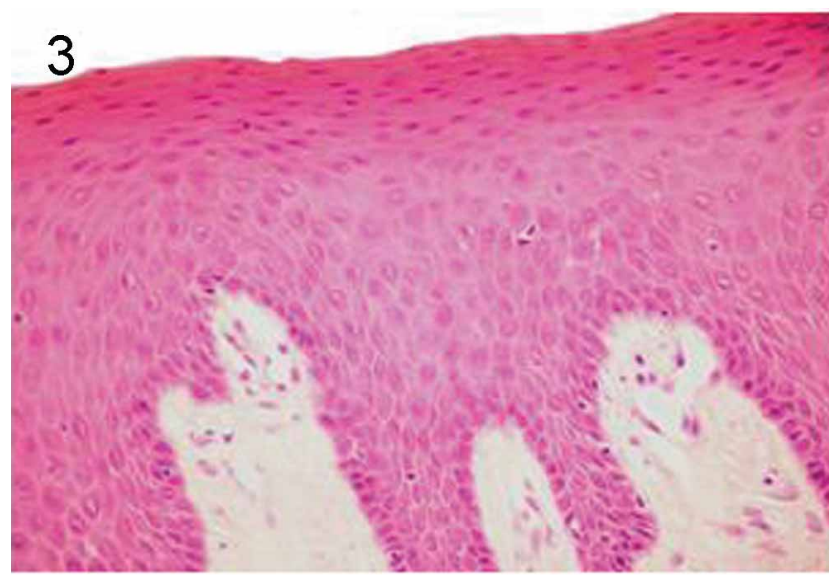

Fig. 3. Hiperplasia fibroepitelial inflamatoria oral (H/E- 400x).

Basados en las informaciones de la literatura, sustentadas por el análisis histomorfológico de este estudio, se sugirie que, a pesar que desde el punto de vista histopatológico ciertas lesiones hiperplásicas de la mucosa

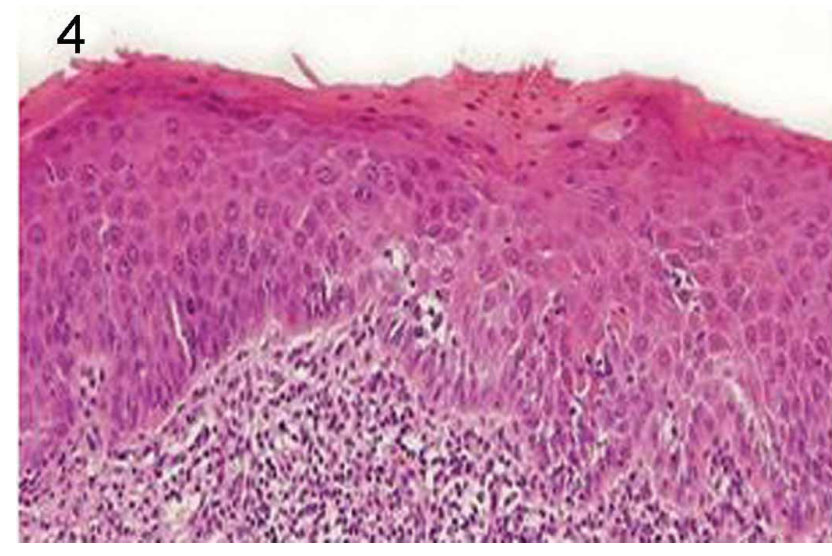

Fig. 4. Displasia epitelial oral leve (H/E - 100x).

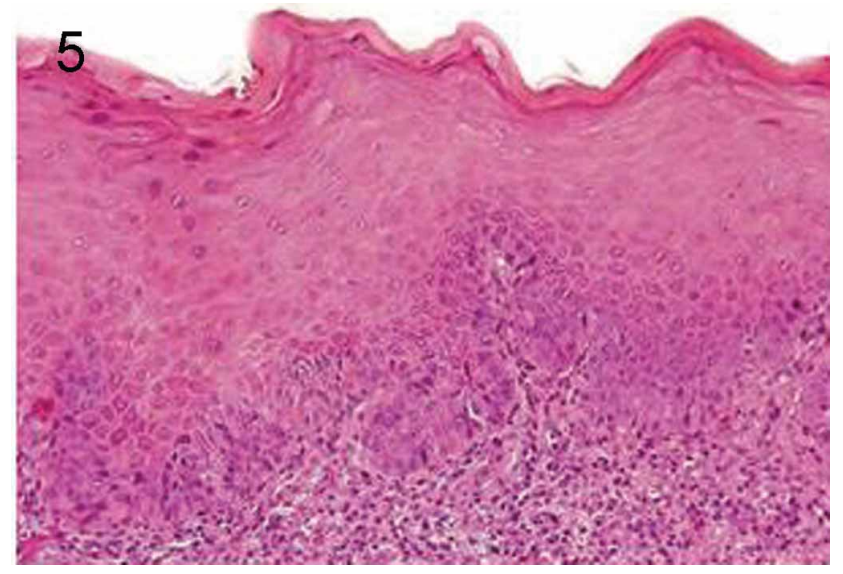

Fig. 5. Displasia epitelial oral moderada (H/E - 100x).

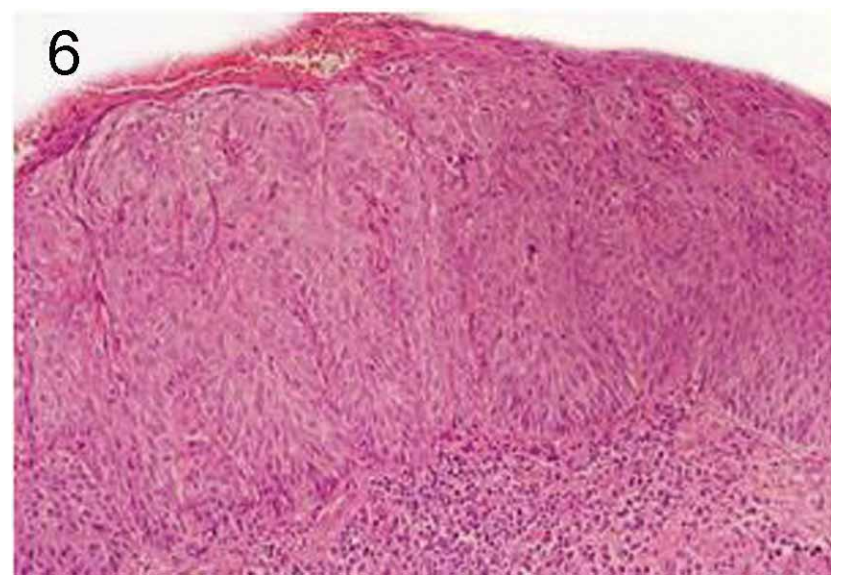

Fig. 6. Displasia epitelial oral intensa (H/E - 100x).

oral puedan exhibir características comúnmente observadas en lesiones con potencial maligno, como las displasias, debemos considerar que muchas de estas alteraciones podrían estar asociadas al proprio proceso inflamatorio donde ac- 
túan mediadores químicos con capacidad de promover alteraciones en la morfología celular y en la organización estructural tisular (Kumar et al.).

Los datos de este estudio concuerdan con los de la literatura en relación a la edad de mayor frecuencia para las DEOs, verificándose una mayor ocurrencia de estas lesiones en pacientes mayores de 50 años (Jaber et al., 1999, 2003). Los autores antes citados, tampoco encontraron diferencia significativa en la ocurrencia de DEO entre los diferentes sexos; sin embargo, al contrario de lo observado en este estudio, ellos verificaron una tendencia a mayor frecuencia de la lesión en el sexo masculino.

Probablemente esos datos controversiales guardan relación con particularidades de la muestra, una vez que el estudio de ellos fue realizado en Europa, donde los hábitos de los pacientes y particularidades del ambiente difieren de los de la muestra analizada en este estudio, pudiendo tener esto alguna influencia en el compromiso de un determinado sexo, de acuerdo con la población y la región geográfica evaluada. Además, podemos destacar el hecho que en Brasil, generalmente los pacientes del sexo femenino, buscan con mayor frecuencia los servicios de salud por lesiones en la cavidad oral, lo cual podría tener alguna relación con la mayor detección de DEOs en las mujeres de la muestra evaluada.

Aún cuando no fue observada diferencia expresiva en la ocurrencia de DEO con relación al color de piel de los pacientes, fue constatado que $44 \%$ de las DEOs ocurrieron en pacientes blancos, estos datos también concuerdan con los de Jaber et al. (2003) quienes verificaron una mayor frecuencia de esa lesión en pacientes de piel blanca.

Considerando la localización de las DEOs, fue observado que la mayoría de estas estaba presente en regiones de mucosa yugal, labio inferior, lengua, paladar y región retromolar, locales que según Küffer \& Lombardi cuando se encuentran expuestos a factores carcinogénicos son más susceptibles al desarrollo de un proceso neoplásico que otras áreas de la mucosa oral. En los estudios de Jaber et al. (2003) y Tosios et al. también fueron observados resultados similares a los de este estudio con relación a la localización de las DEOs.

Es aceptado que la frase "sitios de alto riesgo" para el desarrollo de neoplasias epiteliales en la cavidad oral no está bien fundamentada por evidencias científicas; sin embargo existe la probabilidad de que locales como el piso oral, vientre y bordes de la lengua puedan ser significativamente más susceptibles a la acción de carcinógenos diluidos en la saliva, de que otras regiones de la cavidad oral (Reibel).
Correlacionando el aspecto clínico de las lesiones, a través de los diagnósticos clínicos registrados en las fichas, con el diagnóstico histopatológico de DEO en la muestra analizada en este estudio, no fue verificada diferencia significativa; sin embargo, observamos que la mayoría de los especímenes tuvo diagnóstico clínico de leucoplasia, HFIO, carcinoma y queilitis actínica. Estos datos concuerdan con la literatura, donde se verifica que muchas de las lesiones de aspecto clínico blancuzco, principalmente las leucoplasias, son diagnosticadas histopatológicamente como DEOs (Jaber et al., 2003; Reibel; Tosios et al.).

Según Reibel, el proceso de desarrollo del cáncer oral puede darse por la presencia de una lesión precursora inicial o de una mucosa aparentemente normal, destacándose las leucoplasias entre las lesiones precursoras. Para Tosios et al. el riesgo de transformación maligna de lesiones con potencial neoplásico varía de $4 \%$ a $35 \%$ y este riesgo es directamente relacionado al grado de displasia epitelial presente.

En las DEOs de este estudio, solo un caso tuvo diagnóstico clínico de eritroplasia, el cual ocurrió en mucosa yugal de una paciente de 65 años de edad, siendo diagnosticada, histopatológicamente como DEO leve. Este caso esta en consonancia con la literatura en lo referente a la localización y edad de mayor frecuencia para las eritroplasias; sin embargo con relación al sexo, se observó una discrepancia con la literatura, la cual relata una mayor frecuencia en pacientes del sexo masculino, a pesar de que algunos estudios relatan una tendencia variable entre los sexos (Reichart \& Philipsen, 2005).

Aún habiendo sólo un caso de eritroplasia registrado en este estudio, el diagnóstico histopatológico de DEO leve dado al mismo, nos lleva a concordar con Reichart \& Philipsen al comentar que, aunque sean relativamente raras, si su frecuencia es comparada a la de las leucoplasias, cuando están presentes en sitios de alto riesgo de la mucosa oral, las eritroplasias, generalmente constituyen, histopatológicamente, lesiones displásicas e incluso neoplasias epiteliales propiamente dichas.

El proceso evolutivo de mucosa oral normal para carcinoma epidermoide oral es constituido por la concatenación de múltiples eventos, de allí la justificativa de continuar los estudios para intentar esclarecer cuáles son tales eventos y cual es la participación de cada uno de ellos, en las alteraciones sufridas por la mucosa normal, que pueden preceder a la instalación de una neoplasia propiamente dicha, ya que algunas de las lesiones de la mucosa oral pueden representar fases diferentes de una futura neoplasia epitelial (Küffer \& Lombardi). 
La simple observación de las características morfológicas de las alteraciones de la mucosa oral puede auxiliar el establecimiento del diagnóstico de las lesiones sufridas por esa mucosa; sin embargo, no ofrecen mucha información sobre cuáles serían los eventos que llevarían al establecimiento de una determinada lesión.

En este sentido, por ejemplo, concordamos con Okazaki et al. (2002) al comentar que es difícil diferenciar una DEO severa de un carcinoma in situ, o si la presencia de DEO severa llevaría obligatoriamente a un carcinoma. Desde el punto de vista de un análisis histopatológico con hematoxilina/eosina, abstraer esta información es casi im- posible, a no ser que la lesión sea dejada a su propio curso clínico; sin embargo, éticamente, esta posibilidad es inviable, principalmente tratándose de una lesión que puede poseer un riesgo intrínseco de transformación maligna.

Por lo tanto, basado en la información antes citada, se justifican los estudios con técnicas más elaboradas como la inmunohistoquímica con anticuerpos contra moléculas celulares o de la MEC y mas recientemente, la ejecución de estudios de biología molecular con el objetivo de auxiliar en la búsqueda de información que permita entender la compleja multifactorialidad involucrada en el proceso neoplásico del epitelio de la mucosa oral.

GORDÓN-NÚÑEZ, M. A.; SILVA JÚNIOR F. L.; LUCENA, H. F.; GALVÃO, H. C.; DE SOUZA, L. B. \& PEREIRA PINTO, L. Clinical and histomorphological analysis of normal oral mucosa, oral inflammatory hyperplasia and oral epithelial displasia. Int. $J$. Morphol., 26(2):345-352, 2008.

SUMMARY: The oral cancer development can be from apparently normal mucosa, or from precursory lesions, that can represent different step of oral epithelial carcinogenesis process. This study aimed to analyze morphologically trough the hematoxilina/eosine technique normal oral mucosa, oral fibroepithelial inflammatory hyperplasia and oral epithelial dysplasia (OED) to get more informations about the epithelial alterations that can to precede the installation and evolution of oral cancer. Its was observed that some of the morphologic alterations in the oral fibroepitelial hyperplasia were provably associated to the inflammatory process, where exist the action of chemical mediators with capacity to promote cellular morphologic modifications and alterations of the tissue architecture but not to common alterations of lesions potentially malignant. Correlating the clinical aspect of the lesions with the histopatologic diagnosis of oral epithelial dysplasia its was not observed expressive difference, however the majority of the OED specimens had clinical diagnosis of leucoplasia. Its was concluded that the simple observation of the morphologic characteristics of epithelial alterations in the oral mucosa can to improve the diagnostic of the lesions suffered by this mucosa, however, its not offers much informations about which would be the events that would lead to the establishment of a determined lesion.

KEY WORDS: Oral mucosa; Oral inflammatory hyperplasia; Oral epithelial dysplasia; Histomorphology.

\section{REFERENCIAS BIBLIOGRÁFICAS}

Castellanos, J. L. Displasias y carcinomas de la mucosa bucal. Revista ADM, 59(4):155-6, 2002.

González-Moles, M. A. Lesiones precancerosas de la mucosa oral. RCOE, 2(8):599-617, 1997.

Jaber, M. A.; Porter, S. R.; Gilthorpe, M. S.; Bedi, R. \& Scully, C. Risk factors for oral epithelial dysplasia--the role of smoking and alcohol. Oral Oncol., 35(2):151-6, 1999.

Jaber, M. A.; Porter, S. R.; Speight, P.; Eveson, J. W. \& Scully, C. Oral epithelial dysplasia: clinical characteristics of western European residents. Oral Oncol., 39(6):589-96, 2003.

Kambic, V. Epithelial hyperplastic lesions - a challenging topic in laryngology.Acta Otolaryngol., 527:7-11, 1997.
Küffer, R. \& Lombardi, T. Premalignant lesions of the oral mucosa. A discussion abot the place of oral intraepithelial neoplasia (OIN). Oral Oncol., 38(2):125-30, 2002.

Kumar, V.; Abbas, A. K. \& Fausto, N. Robins e Cotran. Patologia-Bases Patológicas das Doenças. $7^{\mathrm{a}}$ ed. Rio de Janeiro, Elsevier, 2005.

McCullough, M.; Jaber, M.; Barrett, A. W.; Bain, L.; Speight, P. M. \& Porter, S. R. Oral yeast carriage correlates with presence of oral epithelial dysplasia. Oral Oncol, 38(4):391-3, 2002.

Montenegro, M. R. \& Franco, M. Patologia-Processos Gerais. $4^{\mathrm{a}}$ ed. Rio de Janeiro, Editora Atheneu, 1999.

Neville, B. W.; Damm, D. D.; Allen, C. M. \& Boquot, J. E. Patologia Oral e Maxilofacial. $1^{\mathrm{a}}$ ed. Rio de Janeiro, Guanabara Koogan, 2004. pp. 287-96. 
GORDÓN-NÚÑEZ, M. A.; SILVA JÚNIOR F. L.; LUCENA, H. F.; GALVÃO, H. C.; DE SOUZA, L. B. \& PEREIRA PINTO, L. Análisis clínico e histomorfológico de la mucosa oral normal, hiperplasia fibroepitelial inflamatoria oral y displasia epitelial oral. Int. J. Morphol., 26(2):345-352, 2008.

Okazaki, Y.; Tanaka, Y.; Tonogi, M. \& Yamane, G. Investigation of environmental factors for diagnosing malignant potential in oral epithelial dysplasia. Oral Oncol., 38(6):562-73, 2002.

Oliver, R. J.; McDonald, D. G. \& Felix, D. H. Aspects of cell proliferation in oral epithelial dysplastics lesions. J. Oral Pathol. Med., 29(2):49-55, 2000.

Pindborg, J. J.; Reichart, P. A.; Smith, C. J. \& van der Wall, I.. WHO: Histological typing of cancer and precancer of the oral mucosa. $2^{\text {nd }}$ ed. New York, Spring, 1997.

Pinto-Coelho, C. M. \& Zucoloto, S. Hiperplasia fibroepitelial inflamatória da cavidad oral. Revista APCD, 52(5):383-7, 1998.

Reibel, J. Prognosis of oral pre-malignant lesions: significance of clinical, histopathological, and molecular biological characteristics. Crit. Rev. Oral Biol. Med., 14(1):47-62, 2003.

Reichart, P. A. \& Philipsen, H. P. Oral erythroplakia--a review. Oral Oncol., 41(6):551-61, 2005.

Stern, I. R. Membrana mucosa oral. In: Bhaskar, S. N. Histologia e embriologia oral de Orban. $10^{\mathrm{a}}$ ed. São Paulo, Artes Médicas, 1989. pp275-351.

Tosios, K. I.; Kapranos, N. \& Papanicolaou, S. I. Loss of basement membrane components laminin and type IV collagen parallels the progression of oral epithelial neoplasia. Histopathology, 33(3):261-8, 1998.

van der Wall, I.; Schepman, K. P.; Van der Meij, E. H. \& Smeele, L. E. Oral leukoplakia: a clinicopathological review. Oral Oncol., 33(5):291-301, 1997.

Winning, T. A. \& Townsend, G. C. Oral mucosal embryology and histology. Clin. Dermatol., 18(5):499-511, 2000.

Zerdoner, D. The Ljubljana classification - its application to grading oral epithelial hyperplasia. J. Craniomaxillofac. Surg., 31(2):75-9, 2003.
Dirección para correspondencia:

Dr. Leão Pereira Pinto

Programa de Pós-Graduação em Patologia Oral - UFRN

Avenida Senador Salgado Filho 1787

CEP 59056-000

Lagoa Nova, Natal / RN

BRASIL

\section{E-mail: leao@pesquisador.cnpq.br} Ippinto@digi.com.br

Recibido : 19-09-2007

Aceptado: 22-11-2007 